\title{
MODERN FEATURES OF PRICE FORMATION ON THE REACTIVE POWER MARKET
}

\author{
Vladimir Kolibaba ${ }^{1}$, Konstantin ZHabin ${ }^{2}$, Anatolij Kolesnikov ${ }^{3}$, Sergey Filin ${ }^{3}$, Maksim Maksimov ${ }^{3}$, Ona Grazyna \\ Rakauskiene $^{4}$, and Sergey Melnikov ${ }^{3}$ \\ ${ }^{1}$ Ivanovo Power Engineering Institute, 153003, Rabfakovskaya Street, 34, Ivanovo, Russian Federation \\ ${ }^{2}$ Branch of JSC "SO UES" ODU Center, 109074, Kitaygorodsky Lane, 7, block 3, Moscow, Russian Federation \\ ${ }^{3}$ Plekhanov Russian University of Economics, Department of Management, 117997, Stremyanny Lane, 36, Moscow, Russian Federation \\ ${ }^{4}$ Mykolas Romeris University, Ateities st. 20, LT-08303 Vilnius, Lithuania
}

\begin{abstract}
This article discusses the specific features of such a product as reactive power. The degree of reactive power compensation both in the domestic electric power industry and in most industrialized countries is clearly insufficient. The possibilities available in electric grids, at power plants and at consumers of electricity for coordinated regulation of reactive power and voltage levels are not fully used. The main factors influencing the formation of the reactive power market in the electric power industry are revealed. The proposed market is based on the organization and implementation of qualified selection of reactive power suppliers. The scheme of the price bid of reactive power suppliers, developed on the fundament of the cost structure of various types of reactive power suppliers is proposed. Rational regulation of reactive power is currently not considered as an economic problem, but it is one of the main tools for managing the efficiency of EPS. The proposed organizational mechanism of pricing for reactive power and the qualified selection of its suppliers is aimed at determining the range of suppliers of reactive power capable of meeting the demand for it with the least costs, as well as to stimulate investments in the construction of new sources of reactive power.
\end{abstract}

\section{Introduction}

In modern society, the need for energy sources and electric power consumption is constantly increasing. The electric power industry has an important role in the development of the country's economy, and therefore there is a need to ensure the effective use of consumed electric power, maintain the quality of electricity and the required level of reliability of electric power systems (EPS). The urgency of the problem of maintaining the standard parameters of the quality of electricity is also exacerbated in connection with the increase in the proportion of renewable energy sources, which have, dominantly, intermittent mode of operation [1-3].

The control of reactive power (RP) flows in EPS plays an important role in ensuring the minimization of losses in the transmission of electricity, the required parameters of its quality and the reliability of energy supply to consumers. In this regard, effective management of RP flows is extremely relevant and one of the significant factors in ensuring the sustainability of the functioning of modern EPS.

Reactive power is a fraction of the total electrical power expended in electromagnetic processes in electrical equipment. It is an indispensable part of the production, distribution and consumption of active power in the AC system. Reactive power is necessary to ensure the normal operation of most types of electrical equipment: electric motors, welding devices, electric arc furnaces, transformers, etc. [4]. The production of RP is provided by generators (power plants), synchronous compensators, banks of static capacitors, etc. Reactive power parameters affect the voltage levels at network nodes, transmission capacity of power transmission lines, values of active power and electricity losses during their transmission and distribution.

At present, the existing rules for the functioning of the electricity (power) market of the Russian Federation do not ensure the interested and coordinated participation of all entities in this process. There are no mechanisms aimed at attracting investments in the creation of new capacities, the formation of economic signals to market entities, encouraging them to rational consumption and distribution of RP in the EPS. Due to these circumstances, studies aimed at studying the economic nature of this process, the problems of pricing and pricing strategies based on costs, competition and demand, are relevant. There are also problems with the assessment of energy assets and the investment potential of energy companies [5-7]. In many cases, they can be solved with the active development of modern innovative, digital technologies [8,9]. Because of these circumstances, research aimed at examining the economic nature of this process, the problems of pricing 
and price strategies emanating from expenses, competition and demand for energy supply to consumers are relevant [10].

\section{Methodology}

In the framework of the presented work, the authors used the following research methods: 1) at the theoretical level: study and generalization, formalization, analysis and synthesis, induction and deduction, axiomatics; 2) at the experimental-theoretical level: experiment, analysis, modeling, synthesis; 3) at an empirical level: observation, measurement, comparison.

\section{Results and Discussion}

The division of a significant share of electric power facilities between different owners has created additional difficulties in managing the EPS as a single complex. In particular, due to technical features in the EPS, along with active power ("beneficial" for the end consumer), $\mathrm{RP}$ is constantly generated and consumed, which is not spent on performing effective work and, in contrast to active power, cannot be converted into mechanical, thermal, light and other forms of energy. Nevertheless, $\mathrm{RP}$ is an integral and necessary part of the production, transmission and consumption of electrical energy, and it is also necessary to maintain voltage levels at the nodes of the EPS and ensure its stable and economical functioning. Reactive power can be produced and consumed both by power plants and consumers of electrical energy, as well as by individual elements of the EPS (power lines, transformers, etc.), which, according to their principle of action, convert electricity into another type of energy and use a magnetic field (welding equipment, electric motors, induction furnaces, etc.). At the same time, an excessive surplus or deficit of RP significantly reduces the efficiency of the functioning of EPS due to a decrease in the ability of networks to transmit electricity through power lines, generates additional power losses, the instability of EPS emerges, and the quality of electrical energy decreases. In a market economy, RP supply management (its generation and / or consumption) can be carried out at facilities owned by various owners (generating and grid companies, consumers).

There is a certain relationship between the prices for RP and the prices for active capacity, which cannot always be established in the form of a simple functional relationship. In this regard, pricing for RP is acquiring an independent significance, and electricity markets clearly need justification and use of special prices for RP.

Reactive power is a specific product, which follows from its technical and economic characteristics and, above all, from the high localization of its production and consumption (transmitted over long distances with large losses, which significantly reduces the reliability of the EPS).

Reactive power should be considered as a special type of product circulating on the electricity market.
Distinctive features of this product are [11]: 1) overlapping in time the processes of production, distribution and consumption; 2) impossibility of accumulation and storage in sufficient quantities; 3) impossibility to specify in advance the volumes of generation and consumption; 4) relatively large losses (RP cannot be transmitted over long distances, and basically it must be produced close to the place where it is consumed); 5) significant local-territorial character of RP due to an increase in active power losses in the network economy during transmission of RP over long distances; 6) the dependence of the volumes of produced and consumed RP not only on consumers, but also on various elements of the electric power transmission system; 7) compared to active power, there is a stronger dependence of the voltage change on the RP (static characteristic), which significantly affects the regulating effect of voltage; 8) the need to carry out optimization calculations for RP at each section of the network to optimize its distribution, considering seasonality, climatic conditions, day of the week and other factors; 9) territorial discrepancy between the main production centers and regions of consumption of RP; 10) the need for uninterrupted and reliable power supply to consumers, requiring the mandatory creation of RP reserves; 11) significant impact on the social and ecological environment of society (has an indirect character).

Accordingly, one can introduce the main factors influencing the formation of the RP market (figure 1) [12].

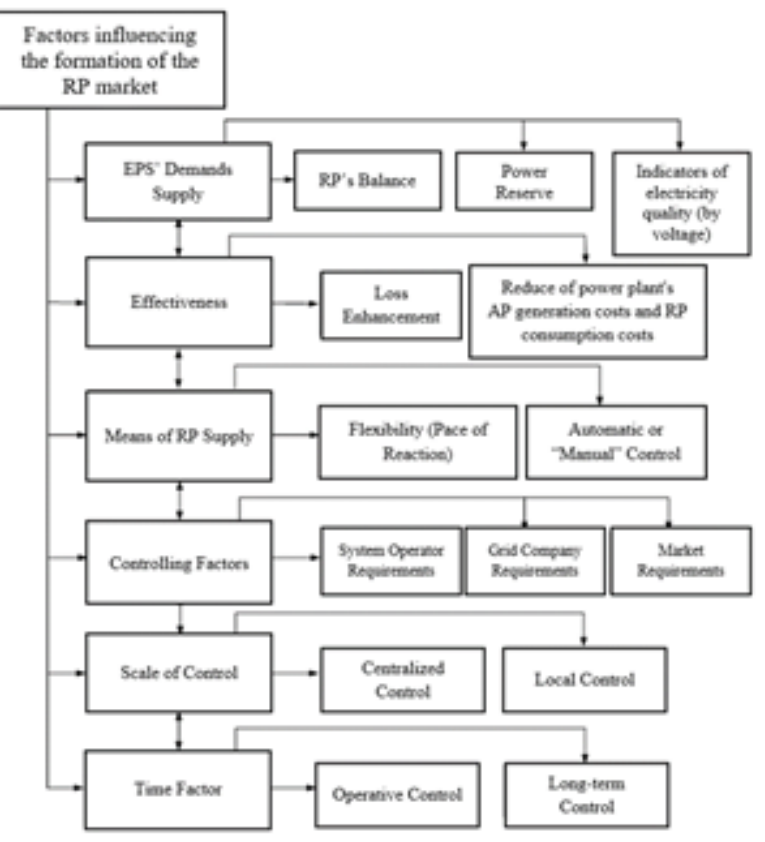

Fig. 1. Factors influencing the formation of the reactive power market.

With spot pricing for RP, the generated prices are characterized by a high degree of volatility. Therefore, in order to eliminate this price volatility, it is proposed that the RP market be long-term, where the System Operator would conduct once a year (or as required) 
qualified selection of RP suppliers. At the same time, it should be noted that ensuring a balance between supply and demand in RP in the long term will not guarantee that supply and demand in RP will coincide in the short term. To protect against this risk, it is possible to provide appropriate economic incentives to RP suppliers [13]. Incentives in this case can be created by introducing rules for the mandatory provision of RP with the subsequent payment of the costs incurred $[14,15]$. This tool can be used if there are problems with market concentration.

Any wholesale market entity that can regulate RP in an electric power system must have the right to enter the RP market. At the same time, all selected RP suppliers should be paid in accordance with their submitted price requests, which in turn should reflect all the expenses incurred related to the supply of RP. Therefore, the main tool used in calculating the price on the RP market should be a price request from RP suppliers, which indicates the desired volumes and prices at which the participant is ready to sell RP. It is therefore important to develop an appropriate price requisition structure.

The structure of the price request of RP suppliers was developed on the basis of the study of the cost structure of RP suppliers. Reactive power can be generated and consumed at very low cost by the equipment of electricity consumers, which does not require the consumption of fuel resources for its operation. It is also generated by power plant generators and is an essential part of the active power generation process. The costs of power plants for the generation or consumption of RP are associated with the costs of maintaining the generating equipment in readiness (fixed costs) and the following variable costs: 1) fuel costs (when the generator is operating in generator mode); 2) the cost of lost profits due to a decrease in the generator's ability to produce active power; 3) active power losses; 4) repair costs due to damage to mechanical parts of the generator due to strong temperature fluctuations (faster aging of some equipment elements due to increased electric current as a result of their heating) and vibrations.

It is proposed to pay for the operation of power plants for the generation (consumption) of RP according to the price order formation scheme shown in figure 2 .

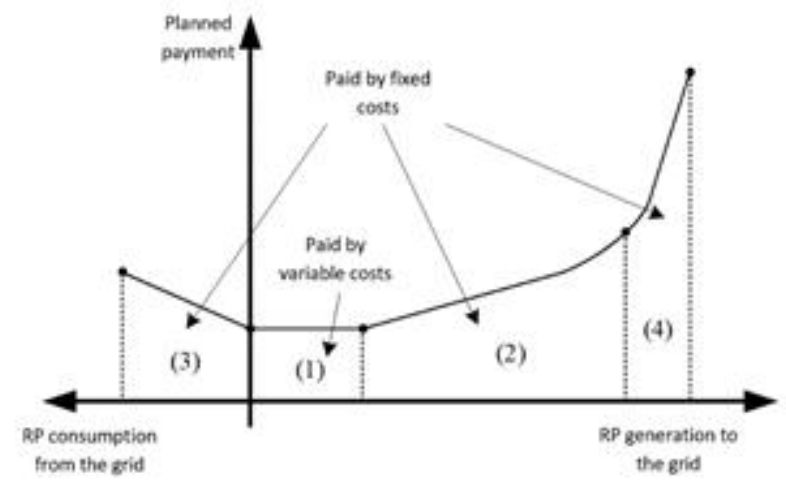

Fig. 2. Factors influencing the formation of the reactive power market.
This scheme is based on the technical features of the operation of synchronous generators and consists of four paid ranges: 1) paid range for maintaining the readiness of the equipment in which the generator generates PM to meet its technological requirements for normal operation, generation of active power (1). In this range, the synchronous generator can work stably, generate active power in its entire available range; 2) paid ranges for generation (2) and consumption (3) of RP. The generator price offer includes payment for the actual generated (consumed) RP; 3 ) paid range for lost profits incurred in the electricity market (4). It arises at the request of the System Operator, when the generator is obliged to reduce the generation of active power in order to increase the generation of RP. This cost of RP reflects the cost of lost profits from the sale of active power (electricity) on the market.

Based on the foregoing and on the basis of the allocated costs of various technologies for regulating RP, the following structure of price bids is proposed: 1 . For power plant generators (figure 2 ):

$$
B_{g}=\left\{\begin{array}{l}
C_{g}, \text { if generator is in the segment }(1), \\
C_{g}^{\text {gen }}, \text { if generator is in the segment }(2), \\
C_{g}^{\text {con }}, \text { if generator is in the segment }(3), \\
C_{g}^{\text {alt }}, \text { if generator is in the segment }(4),
\end{array}\right.
$$

where: $B_{g}$ - value of the price bid of the generator $g$ over generation (consumption) of RP, rubles/MVAr $\cdot \mathrm{h}, C_{g}-$ is the component of the price bid reflecting the fixed costs of the generator $g$, rubles/MVAr; $C_{g}^{\text {gen }}-$ is the component of the price bid, reflecting the variable costs of the generator $g$ associated with the generation of RP, rubles/MVAr $\cdot \mathrm{h} ; C_{g}^{\text {con }}$ - is the component of the price bid, reflecting the variable costs of the generator $\mathrm{g}$ associated with the consumption of RP, rubles/MVAr . $\mathrm{h} ; C_{g}^{a l t}-$ the component of the price bid, reflecting the variable costs of lost profits of the generator $g$, rubles/MVAr $\cdot h$.

2. The expenses of lost profits associated with the supply of RP to power plants is currently being recovered through a balancing market mechanism. This mechanism does not provide appropriate economic signals to RP consumers about its real value and does not encourage them to more responsible consumption of RP [11]. In this regard, it is proposed to reimburse these expenses in the RP market, which should be taken into account in the price applications of power plants.

The variable expenses of power plants are significantly higher than other RP sources, which are associated with active power losses and generator life. At the same time, other PM sources are dominated by fixed expenses, and variable expenses are generally quite low. Therefore, variable costs for these sources in the PM market can be neglected. For these RP control technologies (consumer power plants):

$$
B_{h}=C_{h} \text {, }
$$

where $C_{h}-$ is a constant component of the costs of sources of RP, rubles / MVAr $\cdot h$. 
Figure 3 shows the possible types of quotations of different RP control technologies.

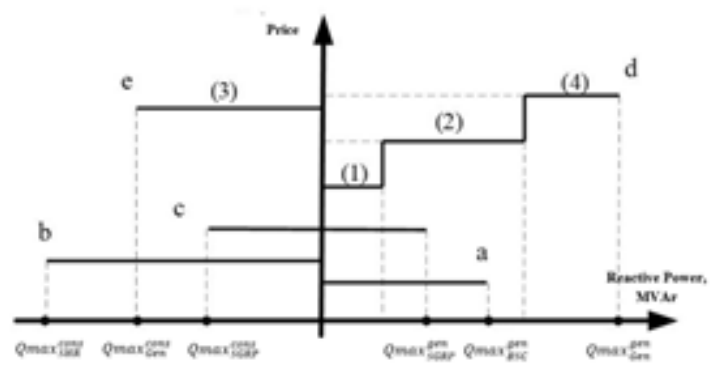

Fig. 3. Possible price requisitions from different reactive power control technologies: a - supplier price bid, Batteries of Static Capacitors (BSC); b - supplier price bid, Shunt Reactors (SHR); c - supplier price bid, Static Thyristor Compensators (STC); d, e - price bids of power plants (presented by piecewise-linear functions).

\section{Conclusion}

1. Reactive power is an integral and necessary part of the production, transmission and consumption of electrical energy, and it is also necessary to maintain voltage levels in the nodes of the EPS and ensure its stable and economical functioning. This is a specific product, the trade of which requires the formation of special methods and pricing tools.

2. Rational regulation of RP is currently not considered as an economic problem, but it is one of the main tools for managing the efficiency of EPS. In all countries of the world, there are currently no full-fledged competitive economic mechanisms to stimulate the commissioning of new sources of RP and pricing for RP, which would form its suppliers a fair price and at the same time, due to its specifics, would send appropriate price signals to the participants of the electric power market for its rational consumption.

3 . The proposed organizational mechanism of pricing for RP and the qualified selection of its suppliers is aimed at determining the range of suppliers of RP capable of meeting the demand for it with the least costs, as well as to stimulate investments in the construction of new sources of RP.

\section{References}

1. A.V. Iunikova, A.M. Karyakin, T.S. Latushkina, V.V. Velikorossov, G.O. Zhanguttina, A.K. Zaharov, Jardcs., 12, 6 (2020)

2. V.V. Velikorossov, A.M. Karyakin, A.S. Tarasova, Intellectual Economics, 13, 2 (2019)

3. A. Tarasova, V. Velikorossov, S. Filin, S. Ibraimova, A. Yakushev, in the collection: E3S Web of Conferences, 1. Ser. "1st International Conference on Business Technology for a Sustainable Environmental System, BTSES 2020" (2020)

4. I.A. Kalinina, V.V. Maslennikov, S.A. Filin, Ecology of industrial production, 13 (2018)
5. V.V. Velikorossov, V.I. Kolibaba, A.A. Ovsyannikov, Analysis of the reliability of the functioning of distribution network companies included in IDGC of Center and Volga Region for 2011-2016, Development of integration processes in the Russian economy, Rusains, Moscow (2018)

6. V.V. Velikorossov, A.M. Karyakin, A.S. Tarasova, Economics and Management: Problems, Solutions, 9, 3 (2019)

7. A.S. Tarasova, Z.H. Dong, V.V. Velikorossov, A.M. Karyakin, N.A. Prodanova, A.V. Kolesnikov, Journal of Advanced Research in Dynamical and Control Systems, 12 (2020)

8. E. Genkin, S. Filin, V. Velikorossov, Z. Kydyrova, K. Anufriyev, in the collection: E3S Web of Conferences, 1. Ser. "1st International Conference on Business Technology for a Sustainable Environmental System, BTSES 2020" (2020)

9. V.V. Velikorossov, A.V. Rechinskiy, L.V. Chernenkaya, S.A. Filin, A.V. Chernenkii, Digital Economy as a Tool for Reducing of Uncertainty in Strategic Managerial Decisions, Proceedings CSIS'19 Eleventh International Scientific and Theoretical Conference "Communicative strategies of Information Society" (2019)

10. D.N. Zemlyakov, S.A. Filin, L.A. Tchaikovskaya, S.V. Khorshikyan, A.I. Yakovlev, Analysis of the reliability of the functioning of distribution network companies included in IDGC of Center and Volga Region for 2011-2016. Development of integration processes in the Russian economy, Rusains, Moscow (2018)

11. V.I. Kolibaba, K.V. ZHabin, Economics and Bulletin of Ivanovo State Energy University, 2 (2017)

12. V.E. Vorotnitsky, V.I. Kolibaba, K.V. ZHabin, Power plants, 5 (2020)

13. S.A. Filin, Insurance and hedging of investment risks (Ankil, Moscow, 2019)

14. T.A. Vaskovskaya, Electricity, 7 (2015)

15. V.V. Velikorossov, A.M. Karyakin, A.S. Tarasova, Economics and Management: problems, solutions, 6, 10 (2018). 